\title{
Canadian Society for Aesthetic Plastic Surgery 41st Annual Meeting
}

\author{
September $19 \& 20,2014$ • Toronto, Ontario \\ Program Chairmen: \\ Dr Sandy Pritchard, President - Dr Nicholas Carr, Vice-President
}

01

\section{FOLLICULAR UNIT EXTRACTION, A COMBINATION OF THE "FUTURE" AND THE "PAST": A SOBER ASSESSMENT WP Unger Toronto, Ontario}

A majority of patients seen by hair restorations surgeon are not appropriate candidates for Follicular Unit Extraction (FUE), whether it is done manually or utilizing Neograft equipment or the Artas robot. This lecture will explain the reasoning behind the preceding statement:

There are two unavoidable truths in hair transplanting:

A. Hairs, at most, survive in their new place only for as long as they would have in their original location.

B. The fringe hair surrounding the area of Male Pattern Baldness gets intermittently narrower and the hair caliber finer for an individual's life time.

Given the above, hair restoration surgeons should obviously be careful to obtain their grafts from areas in the fringe where the hair is most likely to be permanent (as most doctors and most patients with Male Pattern Baldness (MPB) can be assumed to prefer "permanent hair"). However both Neograft and the Artas employ a graft harvesting technique referred to as FUE, in which Follicular Units (FU) are obtained using a small trephine to punch out the FU directly from the scalp. In order to avoid the donor site looking "moth eaten" immediately after surgery or years later when the fringe hair has become finer and sparser, the FU must, in most patients, be spread approximately $4 \mathrm{FU}$ apart. This means that if the patient will require a large number of grafts - in order to adequately treat an average or larger than average area of MPB - some grafts will have to be taken from peripheral areas of the fringe hair, where a higher percentage of hairs are more likely to eventually be lost. In addition, scars in the periphery of the donor area become more easily visible as the hair gets sparser and finer; this becomes even more problematic if all the hair surrounding the graft scars is eventually lost. Photos clarifying and elaborating on these potential problems will be presented in the lecture; interestingly these potential later downsides of FUE parallel those of the old larger round graft harvesting technique employed in the early days of hair transplanting, and were the main impetus to the change to of single scar strip harvesting. (Are we going forward or backwards?)

Another speaker at this meeting will present the very real advantages of the technique of FUE and the two motorized methods that are currently available - Neograft and the Artas robot. However the above noted disadvantages make FUE by any means, less optimal for most patients than the traditional strip harvesting method, in which all grafts are obtained from the most central and densest area of the fringe hair, where the highest percentage of most likely permanent hairs are found.

02

THE ROLE OF LARGE VOLUME FAT GRAFTING IN AESTHETIC BREAST SURGERY - WHERE ARE WE? L Bucky

Philadephia, Pennsylvania, USA
03

\section{ALCL UPDATE \\ W Peters \\ Toronto, Ontario}

Since 1997, reports from the scientific community have suggested a possible association, without causation, between breast implants and anaplastic large cell lymphoma (ALCL). Analysis of these patients has been challenging. Many studies have been under-reported while others have been duplicated. In 2011, the FDA "white paper" analyzed 34 of the 60 cases reported worldwide. All 34 patients had undergone secondary surgery for breast swelling, firmness or pain. ALCL was an incidental finding. Diagnosis of ALCL is made by H\&E histology and immunochemistry for the CD30 marker. ALCL occurred with all types of implants. Subsequent studies have suggested that textured implants may have a greater risk. In all cases, ALCL cells were found in the capsule, in the seroma, or within a mass adjacent to the implant. There was no invasion of the cells beyond the capsule into breast parenchyma. From the FDA study, the risk of developing ALCL after receiving implants appears to be about 1 in 1 million per year. All cases appear to have the ALK-negative marker. ALCL in most of these patients may represent a new entity with less aggressive behavior. In most patients with capsule-confined disease, proper management may prove to be implant removal and capsulectomy. Patients with a distinct mass adjacent to their implant may have a more aggressive clinical course that may become systemic. They may require chemotherapy in addition to implant removal and capsulectomy. All cases of ALCL should be referred to an appropriate specialist and reported to the FDA.

\section{4}

\section{LATE HEMATOMA AFTER BREAST AUGMENTATION}

W Peters, V Fornasier, D Howarth Toronto, Ontario

Late hematoma after breast augmentation is a rare phenomenon with unproven etiology. Most studies have involved individual case reports. The present study describes five patients, who presented with a late unilateral hematoma after receiving bilateral, submuscular, smooth, round, silicone gel implants. There was no history of trauma, steroid use or anti-coagulation. All patients underwent explantation and capsulectomy with extensive histological analysis of all capsules.

RESULTS: All patients presented with a progressive unilateral breast enlargement with at least a doubling of their breast size. This enlargement developed 9, 12, 14, 22, and 38 years after implant insertion. Patients presented for treatment $1,3,4,9$, and 12 months after initial breast enlargement. This allowed an analysis of the sequential events occurring in the capsules. Multiple areas of recent and old hemorrhage were observed, both within the structure of capsules and on their surface. Continuing bouts of recurrent hemorrhage were observed in all patients, even 12 months after initial hematoma development. Microscopic analysis of all capsules demonstrated numerous vessels within the structure of the capsules. The position of these vessels corresponded to the sites of recurrent bleeding. One patient, who underwent a subtotal capsulectomy, continued to form fluid in the implant pocket. Subsequent analysis of that capsule showed persistent hemorrhaging within the structure of the capsule. The differential diagnosis of late unilateral breast enlargement is presented. 
CONCLUSIONS: Late hematomas presented from 9 to 38 years after submuscular breast augmentation. Late hematomas appear to result from multiple recurrent bouts of bleeding from vessels within the structure of the capsules. Breast enlargement develops progressively over time. Failure to remove a capsule can result in continuing bleeding from vessels within the capsule.

\section{5}

\section{ESSENTIALS OF PRIMARY RHINOPLASTY}

\section{B Neu}

Toronto, Ontario

This paper reviews the key elements of a primary rhinoplasty. The thought processes involved in the initial consultation are discussed and conveyed to blueprints. The logic behind various surgical maneuvers is examined, with the view to providing maximum predictability in outcome.

\section{6}

\section{PERSONAL APPROACH TO COMPLEX AND SECONDARY RHINOPLASTIES \\ B Neu}

Toronto, Ontario

While complex rhinoplasties, especially secondaries, can be daunting, an organized, systematic, surgical approach can result in rewarding results. This paper examines some of these challenges, and reviews the surgeon"s evolving approach, especially as it applies to dorsal augmentation.

\section{7}

\section{SURGICAL CORRECTION OF THE SADDLE NOSE DEFORMITY}

O Antonyshyn

Toronto, Ontario

The saddle nose deformity results primarily from a collapse of the middle vault in relation to the nasal tip and root of the nose. However, loss of support in the middle vault has profound effects on adjacent structures. With increasing severity of collapse, there is a progressive rotation and the loss of projection of the nasal tip, retraction of the columella, shortening of the nose, and ultimately, contracture of the nasal soft tissue envelope. The degree and extent of collapse are defined by clinical features which are readily identified on preoperative assessment.

This paper describes a graduated approach to reconstructing saddle nose deformity in relation to the extent of the collapse. Techniques employing cartilage septal extension and columellar grafts in moderate cases, and cantilever bone grafts in more severe cases, to restore an osseocartilagenous supportive framework, will be demonstrated. Methods for further contour refinement and augmentation using dorsal and columellar grafts comprised of fascia or acellular dermis, with or without diced cartilage, will be described. Finally, in cases of extreme collapse, measures to release contracted tissues and restore nasal length will be illustrated.

\section{8}

\section{ABDOMINOPLASTY - 15 YEARS LATER, 15 THINGS DONE DIFFERENTLY}

\section{F Eaves III}

\section{Charlotte, North Carolina, USA}

Despite a long history, abdominoplasty is a procedure that continues to evolve on several fronts. Patient scar positioning preferences change through time, affecting force vectors and closure technique. New forms of perioperative pain control allow reduction in narcotic requirements and improved early postoperative mobility. Various strategies in degree of undermining and combinations of excision with liposuction allow customization of procedures to individual patient needs and preferences. The use of progressive tension or quilting sutures has eliminated the needs for postoperative drains. An increased awareness of venothromobembolic risks in abdominal contouring surgery has also lead to more aggressive prophylaxis regimens. Fifteen different strategies incorporated into practice over the past 15 years highlight the mutually supportive nature of these various changes in current abdominoplasty techniques.

\section{9}

\section{EVOLUTION, BEAUTY \& THE SURGEON}

F Eaves III

Charlotte, North Carolina, USA

The desire for beauty is both innate to and ubiquitous in humans, and based on tens of millions of years of biological, social, and psychological evolution. Beauty provides significant economic, social and mating benefits. Exploring the deep routed mechanisms that drive the quest for beauty provides insights into the both the conscious and unconscious motivations of aesthetic surgery patients and the impact that these changes have upon their lives. Such understanding also gives us clues to psychological reactions to surgery and hints both toward new strategies in patient screening, expectation setting, and postoperative management.

\section{0}

\section{THE K-Z PEXY: BELT AND SUSPENDERS. A NOVEL METHOD TO MANAGE HORIZONTAL LAXITY IN THE LOWER EYELID \\ N Carr}

Vancouver, British Columbia

This paper describes a novel procedure that combines lateral canthopexy with a lateral wedge resection in order to decrease the risk of lower lid malposition in the setting of lower blepharoplasty in patients with horizontal laxity. The author reviews previously described procedures for management of lower lid laxity. The new technique is explained. A series of 22 cases and their outcomes are reviewed.

\section{1}

\section{ABDOMINOPLASTY AND UMBILICAL HERNIA: CONCOMITANT REPAIR USING THE VENTRALEX HERNIA PATCH}

\section{R Neinstein, DA Matarasso, DL Abramson \\ Toronto, Ontario}

BACKGROUND: Patients requesting abdominoplasty often have concomitant umbilical hernias and may request simultaneous treatment. The vascularity of the umbilicus is potentially at risk during these combined procedures. OBJECTIVE: In this study, the authors present a novel technique for treating umbilical hernias at the time of abdominoplasty surgery using the Ventralex ${ }^{\mathrm{TM}}$ hernia patch (Bard, Inc., Covington, GA).

METHODS : A total of 11 female patients with a mean age of 39.4 years (range, 28 to 51 years) undergoing abdominoplasty with umbilical hernia repair with the Ventralex ${ }^{\mathrm{TM}}$ patch were included.

RESULTS: The mean BMI was $27.6 \mathrm{~kg} / \mathrm{m}^{2}$ (range, 20 to $34 \mathrm{~kg} / \mathrm{m}^{2}$ ). No vascular compromise of the umbilicus was seen. The hernia repair did not alter the abdominoplasty results. One patient had transient umbilical swelling post-operatively that resolved at 6 months post-operative.

CONCLUSIONS: We present a series of umbilical hernia repairs in abdominoplasty patients utilizing a minimal access incision via the rectus fascia and the Ventralex ${ }^{\mathrm{TM}}$ patch that is fast and reliable and does not compromise vascularity to the umbilicus.

\section{2}

AN ANALYSIS OF THE RELATIONSHIP BETWEEN EYEBROW SLOPE AND WRINKLE PATTERNS IN THE PERI-ORBIT AND FOREHEAD REGIONS

\section{T DeLyzer, A Yazdani}

London, Ontario

PURPOSE: To characterize changes in the wrinkle pattern of the peri-orbit and forehead regions, and to correlate these with changes in eyebrow slope. METHOD: Standardized AP facial photographs of 100 women aged 20-80 at rest were analyzed. Eyebrow slope was calculated using the height at the medial limbus and highest point. Wrinkle number and severity at the medial forehead (MF), lateral forehead (LF), glabella (GL), and lateral orbit (LO) were graded using a validated wrinkle severity score. The average number and severity at each site was compared across and within groups (decade of age) using the Kruskal-Wallis test. The Mann-Whitney U-test was used to identify significantly different pairings. Similar analysis 
was conducted across and within groups divided by incremental increases in slope.

RESULTS: With increasing age there is a statistically significant differential increase in number and severity of wrinkles, with MF and LF plateauing at the $5^{\text {th }}$ decade, and LO and GL continuing to increase in older decades. The analysis showed that with decreasing slope there was a significant increase in LO number and severity, and this increase was significantly greater compared to MF and LF.
CONCLUSIONS: With aging there is a distinct pattern of wrinkle changes, with a differential increase of LO and GL wrinkles. Eyebrow slope flattening with aging beyond the $5^{\text {th }}$ decade is significantly correlated with increased LO wrinkle severity and number. This could be explained by unopposed orbicularis oculi action at the lateral brow. This information can help us to improve brow rejuvenation techniques.

\section{AUTHOR INDEX TO ABSTRACTS / INDEX DES AUTEURS DES RÉSUMÉS}

A

Abramson DL

Antonyshyn $\mathrm{O} \ldots$

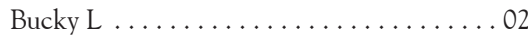

$\mathrm{C}$

Carr N

$\mathrm{D}$

DeLyzer T

\section{$\mathrm{E}$}

Eaves, III F.

Fornasier $\mathrm{V}$

Howarth D

$\mathrm{M}$

Matarasso DA
$\mathrm{N}$

Neinstein R .................... 11

Neu B.................. 05, 06

P

Peters WP. $.03,04$

$\mathrm{U}$

Unger WP .01

$\mathrm{Y}$

Yazdani A..................... 12 